\title{
Sox2 Level Is a Determinant of Cellular Reprogramming Potential
}

\author{
Dong Wook Han ${ }^{1,2^{\star}}$, Natalia Tapia ${ }^{3 \odot}$, Marcos J. Araúzo-Bravo ${ }^{3}$, Kyung Tae Lim ${ }^{1}$, Kee Pyo Kim ${ }^{3}$, Kinarm \\ Ko ${ }^{1,2}$, Hoon Taek Lee ${ }^{1}$, Hans R. Schöler ${ }^{3,4}$
}

1 Department of Stem Cell Biology, School of Medicine, Konkuk University, Seoul, Republic of Korea, 2 Institute of Functional Genomics, Konkuk University, Seoul, Republic of Korea, 3 Department of Cell and Developmental Biology, Max Planck Institute for Molecular Biomedicine, Münster, Germany, 4 University of Münster, Medical Faculty, Münster, Germany

\begin{abstract}
Epiblast stem cells (EpiSCs) and embryonic stem cells (ESCs) differ in their in vivo differentiation potential. While ESCs form teratomas and efficiently contribute to the development of chimeras, EpiSCs form teratomas but very rarely chimeras. In contrast to their differentiation potential, the reprogramming potential of EpiSCs has not yet been investigated. Here we demonstrate that the epiblast-derived pluripotent stem cells EpiSCs and P19 embryonal carcinoma cells (ECCs) exhibit a lower reprogramming potential than ESCs and F9 ECCs. In addition, we show that the low reprogramming ability is due to the lower levels of Sox2 in epiblast-derived stem cells. Consistent with this observation, overexpression of Sox 2 enhances reprogramming efficiency. In summary, these findings suggest that a low reprogramming potential is a general feature of epiblast-derived stem cells and that the Sox 2 level is a determinant of the cellular reprogramming potential.
\end{abstract}

Citation: Han DW, Tapia N, Araúzo-Bravo MJ, Lim KT, Kim KP, et al. (2013) Sox2 Level Is a Determinant of Cellular Reprogramming Potential. PLoS ONE 8(6): e67594. doi:10.1371/journal.pone.0067594

Editor: Maurizio Pesce, Centro Cardiologico Monzino, Italy

Received November 13, 2012; Accepted May 23, 2013; Published June 18, 2013

Copyright: ( 2013 Han et al. This is an open-access article distributed under the terms of the Creative Commons Attribution License, which permits unrestricted use, distribution, and reproduction in any medium, provided the original author and source are credited.

Competing interests: The authors have declared that no competing interests exist.

*E-mail: dwhan@konkuk.ac.kr

๑) These authors contributed equally to this work.

\section{Introduction}

Pluripotency has been recently classified into two distinct states namely, naïve and primed pluripotency [1-3]. The ground naïve pluripotent state refers to cells, such as embryonic stem cells (ESCs), that can form teratomas and contribute to chimeras. In contrast, primed pluripotency occurs in cells, such as epiblast stem cells (EpiSCs), that can form teratomas but can rarely form chimeras [1-3]. Furthermore, leukemia inhibitory factor (LIF) but basic fibroblast growth factor (bFGF) and Activin are required to maintain self-renewal in the naïve and primed pluripotent state, respectively. In female cells, both $X$ chromosomes remain activated in the naïve ESCs while one chromosome is randomly inactivated in the primed EpiSCs [1-3]. However, in spite of these differences, the transcription factors Oct4, Sox2, and Nanog are crucial components of the regulatory circuit in both pluripotency states [4-6]. Moreover, recent reports have confirmed that Oct4 and Sox2 together with Klf4 and c-Myc can induce naïve or primed pluripotency in somatic cells depending on the applied culture conditions [7-9].

P19 is an embryonic carcinoma cell (ECC) line derived from a 7.5 days post coitum (d.p.c.) embryo that was transplanted into the testis [10]. P19 ECCs maintain a male euploid karyotype and can differentiate into all three germ layers, indicating that they are pluripotent [10]. Although P19 ECCs, EpiSCs and ESCs present similar Oct4 expression levels, P19 ECCs and EpiSCs express lower levels of Nanog than ESCs $[11,12]$. Furthermore, P19 ECCs also share other similarities with EpiSCs, such as the preferentially use of the Oct4 proximal enhancer [13]. In contrast to P19 ECCs, F9 ECCs and ESCs preferentially use the Oct4 distal enhancer [13]. In addition, F9 ECCs showed levels of Nanog expression similar to those observed in ESCs. These observations suggest that P19 and F9 ECCs resemble different pluripotent states, a feature that has been successfully used in cell fusion reprogramming experiments to decipher the mechanisms underlying cellular pluripotency and reprogramming [14,15].

In the current study, we investigated the relationship between the pluripotency state and the reprogramming potential. To this end, we used a cell fusion protocol in which distinct pluripotent cell types were used as fusion partners. We found that EpiSCs and P19 ECCs typically exhibit a lower reprogramming potential than ESCs and F9 ECCs respectively, demonstrating that cell types presenting naïve pluripotency have a higher reprogramming potential. We also observed that 
the overexpression of Sox2, which is expressed in lower levels in EpiSCs and P19 ECCs compared with ESCs and F9 ECCs, leads to a dramatically increased reprogramming capability in EpiSCs and P19 ECCs. These findings suggest a close relationship between the pluripotency state and the reprogramming capacity, with Sox2 levels playing a determinant role on the reprogramming potential.

\section{Results}

\section{EpiSCs exhibit a low reprogramming potential}

ESCs and EpiSCs exhibit features of pluripotency, as evidenced by the ability to differentiate into cell types of all three germ layers [1,3]. Though previous reports have thoroughly characterized the potential of ESCs to reprogram somatic cells using cell fusion [14,15], the reprogramming potential of EpiSCs have not been assessed yet. Therefore, we first compared the reprogramming potential of EpiSCs and ESCs after each had been fused with neomycin-resistant NSCs. Following neomycin selection of the fusion hybrids for one week, the rate of colony formation was determined using AP staining. While fusion of NSCs with ESCs led to the production of many viable fusion hybrid colonies, no viable colonies had formed after the fusion of NSCs with EpiSCs (Figure 1A). Thus, we next tried to elucidate the mechanism underlying the extremely low reprogramming potential of EpiSCs. As EpiSCs and human ESCs grow under very similar culture conditions and need to be passaged as small cell clumps, not as single cells, we postulated that the reprogramming efficiency of EpiSCs may be difficult to quantify by using the cell fusion protocol, which requires complete dissociation of EpiSCs into single cells $[1,3,14]$. To increase the survival rate of the completely dissociated EpiSCs, we repeated the fusion experiment in the presence of ROCK inhibitor (Y-27632), which inhibits apoptosis and thus allows human ESCs to be grown as single cells [16]. A 3.6-fold increase in the number of EpiSCs colonies was observed in the presence of the ROCK inhibitor after complete dissociation into single cells (Figure $1 \mathrm{~B}$ and $1 \mathrm{C}$ ). We next fused EpiSCs with NSCs in the presence of the ROCK inhibitor and subsequently observed a few AP-positive fusion hybrid colonies (Figure 1A). These results indicate that EpiSCs indeed have a reprogramming potential ability, although it is extremely low in comparison to that of ESCs. These results suggest that although EpiSCs are pluripotent stem cells, their reprogramming potential differs from that of ESCs.

\section{Low reprogramming potential is a general feature of epiblast-derived pluripotent stem cells}

Next, we tried to exclude that the extremely low reprogramming potential of EpiSCs was due to low survival after dissociation, even in the presence of the ROCK inhibitor. To this end, we investigated the reprogramming potential of P19 ECCs since P19 ECCs and EpiSCs exhibit similar features $[10,13,17]$. P19 ECCs can be dissociated into single cells without affecting survival, thus cell fusion experiments using P19 ECCs could be used as a model to test whether the low reprogramming potential of EpiSCs is a general feature to all

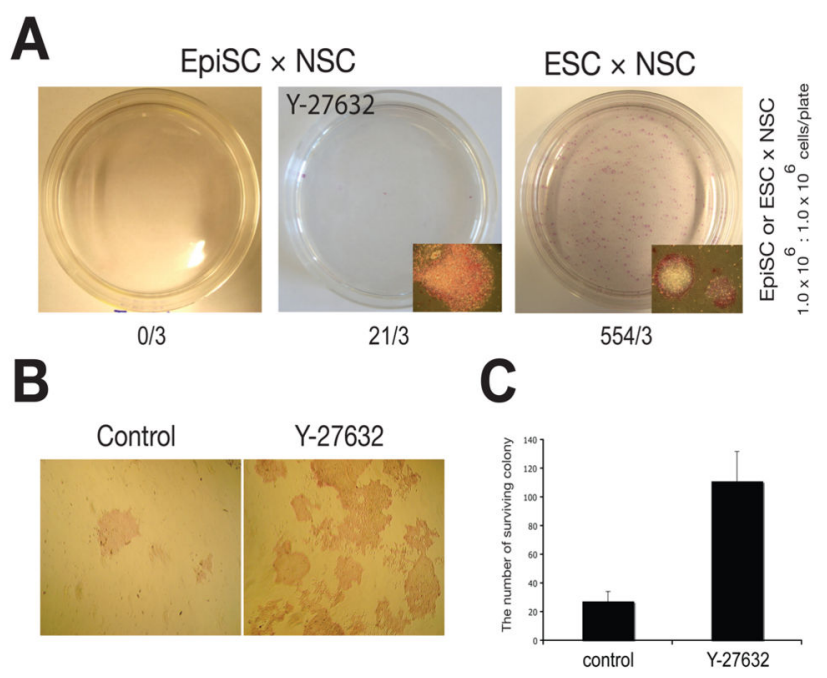

Figure 1. EpiSCs exhibit a low reprogramming potential. (A) Reprogramming potential of EpiSCs and ESCs was determined by comparing the colony-forming rate of the fusion hybrids between NSCs and EpiSCs or ESCs, respectively, in the absence or presence of the ROCK inhibitor $(\mathrm{Y}-27632)$. Data is represented as the number of total colonies from 3 different experiments. (B and $\mathbf{C}$ ) EpiSCs completely dissociated by trypsinization were cultured in the presence or absence of the ROCK inhibitor, and the survival rate was assessed by counting AP-positive fusion hybrid colonies. Data is represented as mean +/- SEM.

doi: 10.1371/journal.pone.0067594.g001

epiblast-derived pluripotent cells or specific to EpiSCs. As control, we used F9 ECCs that resemble ESCs [17]. We compared the reprogramming potential of F9 ECCs with that of P19 ECCs after cell fusion with NSCs containing a green fluorescent protein (GFP) reporter gene driven by the Oct4 promoter. The reprogramming potential was evaluated by monitoring the time required to activate the Oct4-GFP reporter gene expression from the reprogrammed somatic genome. Consistent with our previous findings [14], Oct4-GFP-positive cells were first detected in F9 fusion hybrids within 2 days postfusion. However, Oct4-GFP expression became activated in P19 ECCs within at least 7 to about 9 days after fusion (Figure 2A). Moreover, while fusion of NSCs with F9 ECCs resulted in the demethylation of the Oct4 regulatory regions within 2 days post-fusion [14], the Oct4 proximal enhancer retained its methylated state in P19 fusion hybrids even 1 month postfusion (Figure $2 \mathrm{~B}$ ). $\mathrm{X}$ chromosome reactivation is a well-known epigenetic event occurring after somatic cell genome reprogramming that can be monitored using fluorescence in situ hybridization (FISH) against the Xist/Tsix RNA. In somatic cells, the inactive $X$ chromosome presents a large FISH signal (Xi) while the active $X$ chromosome does not present any signal (Xa). However, both $X$ chromosomes from female ESCs and the single $X$ chromosome from the male ESCs are active but present a pinpoint FISH signal (Xã), suggesting a basal $X i s t / T s i x$ transcription that is specific for the naïve pluripotent state [18]. Interestingly, $X$ chromosome reactivation was 
A

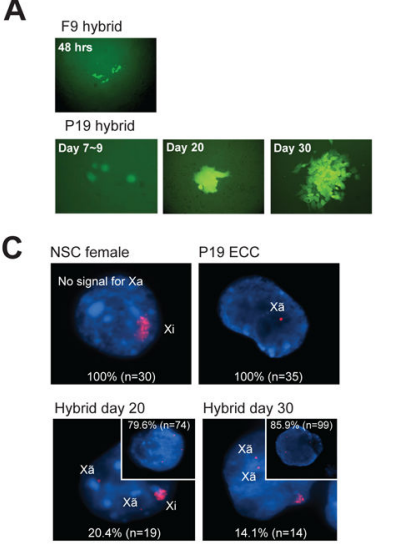

B
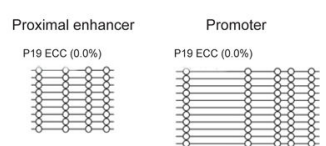

NSC (62.5\%)

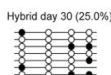

yybrid day $30,25.0 \%$
$8=8=8=$
$8=8=$
$8=8=8:$
$8=8=$

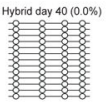

Nsc (42.8\%)
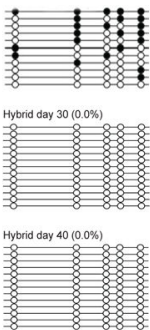

Figure 2. Low reprogramming potential of mouse epiblastderived pluripotent stem cells. (A) Reprogramming potential of F9 and P19 ECCs was determined by monitoring the time required to reactivate the Oct4-GFP transgene in the reprogrammed somatic genome. (B) DNA methylation state of the Oct4 regulatory regions (promoter and proximal enhancer) in P19 hybrids was analyzed using the bisulfite sequencing method. Open and filled circles indicate unmethylated and methylated CpGs, respectively. (C) X chromosome state was determined by Xist/Tsix RNA FISH. DNA was counterstained with 4,6-diamidino-2-phenylindole (DAPI; blue). Xist/Tsix RNA was detected as a large signal in inactive $X$ chromosome $(X i)$, a pinpoint signal in active $X$ chromosome (Xã; pluripotent cell type), and no signal in active $X$ chromosome ( $\mathrm{Xa}$; somatic cell type). In P19 hybrids, the large signal (typical Xi) of female NSCs gradually changed into pinpoint signals, indicating that the $X$ chromosome of the somatic cell genome had been reactivated as in pluripotent $\mathrm{P} 19$ ECCs.

doi: 10.1371/journal.pone.0067594.g002

completed in F9 hybrids within 1 week of fusion [19], but not completed in P19 hybrids even at 1 month post-fusion since a large Xi signal can be still detected in $14.1 \%$ of the hybrids (Figure 2C). Collectively, these findings demonstrate that a very low reprogramming potential of both EpiSCs and P19 ECCs appears to be a general feature of mouse epiblastderived pluripotent cells.

\section{Low levels of Sox2 are responsible for the low reprogramming potential of epiblast-derived pluripotent stem cells}

Although OCT4 protein levels are similar in ESCs and EpiSCs, SOX2 and NANOG levels are lower in EpiSCs than in ESCs as described previously [11]. In agreement with this observation, Oct4 expression was similar in the cell lines tested (ESCs, and F9 and P19 ECCs) but Nanog and Sox2 expression was slightly lower in P19 ECCs than in F9 ECCs, as determined by real-time RT-PCR (Supplementary information, Figure S1A). Low SOX2 levels were confirmed by Western blot (Figure 3A). To assess the effect of Sox2 expression on genomic reprogramming, we first overexpressed Sox2 in P19 ECCs, and confirmed the increase in Sox2

expression by measuring mRNA and protein levels (Supplementary information, Figure S1B and Figure 3A), and then fused these Sox2-overexpressing P19 ECCs, which formed colonies with a slightly compact shape (Supplementary information, Figure S1C), with NSCs. Following neomycin selection, Sox2-overexpressing P19 ECCs (P19-Sox2) led to activated Oct4-GFP transgene expression within 72 hrs postfusion (Figure 3B). Considering that wild-type P19 ECCs activate Oct4-GFP transgene expression after only at least 1 week post-fusion (Figure 2A), it appears likely that delayed reprogramming of $\mathrm{P} 19$ hybrids could be rescued by Sox2 overexpression. After neomycin selection for 1 week, Sox2overexpressing P19 hybrids (P19-Sox2 hybrids) showed a 1.5fold increase in the number of AP-positive hybrid colonies, indicating that Sox2 overexpression led to an increase in the colony formation rate (Figure $3 \mathrm{C}$ ). Moreover, while the proportion of Oct4-GFP-positive reprogrammed cells in the control P19 hybrids was $0.01 \%$, the reprogramming rate in the $\mathrm{P} 19-$ Sox 2 hybrids was $0.2 \%$, representing an approximate 20 fold increase due to Sox 2 overexpression (Figure 3D). These P19-Sox2 hybrids exhibited demethylation of the Oct4 regulatory regions (Figure 3E) earlier than P19 hybrids as well as almost complete $\mathrm{X}$-chromosome reactivation by day 20 post-fusion (98.4\%) (Figure $3 F$ ), versus the less complete $X$ chromosome reactivation $(85.9 \%)$ and delayed demethylation on the Oct4 proximal enhancer element in control P19 hybrids at 1 month post-fusion (Figure 2B and 2C). In summary, these findings suggest that Sox 2 is a key factor in cell fusionmediated reprogramming and that Sox2 overexpression restores the reprogramming potential of P19 ECCs to a level similar to that of F9 ECCs.

\section{The role of Sox2 in reprogramming is not restricted to epiblast-derived pluripotent stem cells}

To investigate whether the role of Sox2 in cell fusionmediated reprogramming is also conserved in non-epiblastderived pluripotent stem cells, we overexpressed Sox2 into F9 ECCs that express higher levels of Sox2 than P19 ECCs (Figure 3A). Similarly, Sox2-overexpressing F9 (F9-Sox2) hybrids (Figure 4A) exhibited an increased colony-forming rate (Figure 4B) and a 12-fold increased reprogramming rate compared with the control F9 hybrids (Figure 4D). Interestingly, the overexpression of Sox 2 in F9 ECCs and F9 hybrid cells resulted in loss of the typical colony morphology and acquisition of a compact ESC-like colony morphology (Figure 4A and 4C), as observed with some clones of Sox2overexpressing P19 cells (Supplementary information, Figure $\mathrm{S} 1 \mathrm{C})$. Therefore, the positive effect of Sox2 on somatic cell genome reprogramming occurs irrespective of the pluripotent stem cell type used in the fusion process.

\section{Sox2 overexpression boosts the low reprogramming potential of EpiSCs}

Although the epiblast-derived stem cell P19 ECCs have a lower reprogramming potential than F9 ECCs and ESCs, P19 ECCs can still reprogram somatic cells with relatively higher efficiency than EpiSCs (Figure 1A). The mRNA levels of Sox2 and Nanog are similar in ESCs and EpiSCs, as shown by 
A

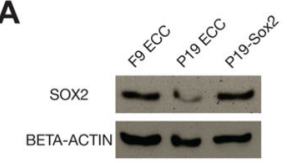

B

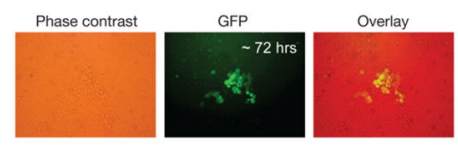

C

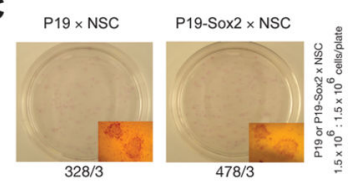

D

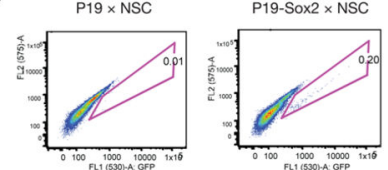

E

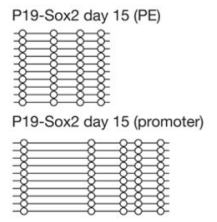

$\mathbf{F}$
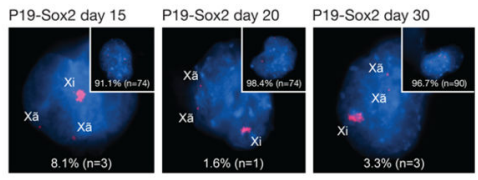

Figure 3. Low levels of Sox2 are responsible for the low reprogramming potential of P19 ECCs. (A) After Sox2 overexpression, SOX2 protein levels were assessed by Western blot. BETA-ACTIN was used as a loading control. (B) The timing of Oct4-GFP activation was monitored under fluorescence microscopy in P19 fusion hybrid cells overexpressing Sox2. (C and D) The colony-forming rates (C) and reprogramming rates (D) were determined by AP staining and FACS, respectively. Data is represented as the number of total colonies from 3 different experiments. (E) The reprogramming patterns of the Oct4 regulatory regions (proximal enhancer and promoter) were analyzed in P19-Sox2 hybrids on day 15 post-fusion by bisulfite sequencing. Open and filled circles indicate unmethylated and methylated $\mathrm{CpGs}$, respectively. (F) X chromosome state in P19-Sox2 hybrids was determined by Xist/Tsix RNA FISH on days 15, 20, and 30 post-fusion. DNA was counterstained with DAPI (blue). Each panel shows the percentage of non-reprogrammed cells, depicting an $\mathrm{X}$ chromosome state consistent with the somatic cell type (a large signal), while the small window shows the percentage of reprogrammed cells, whose $X$ chromosome state is consistent with the pluripotent cell type (three pinpoint signals).

doi: 10.1371/journal.pone.0067594.g003

microarray [3]. Surprisingly, SOX2 and NANOG protein levels are lower in EpiSCs, as determined by Western blot (Figure 5A). To assess whether the extremely low reprogramming potential of EpiSCs is due to low SOX2 protein levels, we overexpressed Sox2 in EpiSCs (Epi-Sox2), as confirmed by increased SOX2 protein levels in EpiSCs (Figure 5A). NANOG levels were also increased, and nearly comparable to ESC levels (Figure 5A). We next investigated whether Sox2 overexpression could also rescue the extremely low reprogramming potential of EpiSCs, as observed for F9 and P19 hybrid cells. To this end, we fused the Epi-Sox2 cells with NSCs (Figure 5A and 5B) in the presence of the ROCK inhibitor. While control EpiSCs produced a few hybrid colonies, Epi-Sox2 cells produced about 5 to 6 times more hybrid colonies (Figure 5B). We next evaluated whether the EpiSC fusion hybrid cells had undergone successful reprogramming. First, we analyzed the gene expression profile of 5 hybrid lines, which were randomly picked from Epi-Sox2 hybrid cell colonies (Figure 5C). As expected, Epi-Sox2 hybrids exhibited a gene expression pattern consistent with successful reprogramming into an EpiSC state. After fusion, there was upregulation of the pluripotency marker genes Oct4 and Nanog, with levels comparable to those of EpiSCs, and of the EpiSC markers Fgf5 and $T$. The control hybrids also showed gene expression patterns similar to those of Epi-Sox2 hybrid cells (data not shown). We next analyzed the epigenetic reprogramming of both control and Epi-Sox 2 hybrid cells by monitoring the DNA methylation state of the Oct4 and Nanog regulatory regions in a time-course manner. While the Nanog and the endogenous Oct4 promoter regions were highly methylated in NSCs, both regions were completely demethylated in control and Epi-Sox2 hybrids within 10 days post-fusion (Figure 5D), indicating that the reprogramming potential of EpiSCs is very low, but once EpiSCs are fused with somatic cells, they can induce normal reprogramming in somatic cells. X-chromosome reactivation was also analyzed in both $X X$ and $X Y$ EpiSC hybrids. EpiSCs are supposed to show the somatic cell forms of the $X$ chromosome (one large signal in $X X /$ no signal in $X Y$ ). As expected, $X X$ EpiSCs have an inactive $X$ chromosome (Figure $5 \mathrm{E}-\mathrm{a})$. Surprisingly, almost half of the XY EpiSCs (44\%) have the pluripotent cell form of the $X$ chromosome (Xã), as they showed only one pinpoint signal, a common characteristic of $X Y$ ESCs (Figure 5F-a). Sox2-overexpressing EpiSCs (XX and $X Y$ ) showed the same $X$ chromosome state as control EpiSCs (Figures 5E-c and 5F-C). XX hybrids from both EpiSCs and EpiSox2 hybrid cells exhibited a non-reprogrammed $X$ chromosome pattern, as they showed two inactive (large signals) and two active (no signals) $X$ chromosomes, indicating that XX EpiSCs have no X-chromosome reactivating potential (Figures $5 \mathrm{E}-\mathrm{b}$ and $5 \mathrm{E}-\mathrm{d}$ ). On the other hand, approximately $38 \%$ of XY EpiSC hybrids showed evidence of X-chromosome reactivation, as the $X$ chromosomes of XX NSCs (a large and no signal) were reprogrammed into two or three pinpoint signals within 7 days post-fusion (Figure 5F-b). However, the rest of the XY EpiSC hybrids (61\%) still exhibited the nonreprogrammed NSC X-chromosome pattern (Xi). Thus, only a subpopulation of $\mathrm{XY}$ EpiSCs is $\mathrm{X}$-chromosome reactivating competent (Figure 5F-b). Unexpectedly, we could not detect the inactivated NSC X-chromosome (Xi) in XY Epi-Sox2 hybrids within 7 days post-fusion, indicating that Sox2 overexpression enhances the $X$ reactivation potential of the $X Y$ EpiSCs (Figure 5F-d). Previously, we have reported that EpiSC cultures are heterogeneous and comprise two distinct subpopulations, Oct4-GFP-positive and -negative EpiSCs, which are in a state of dynamic equilibrium [11]. Oct4-GFPpositive and -negative EpiSCs correspond to in-vivo epiblasts of an early and late developmental stage, respectively, in terms of gene expression patterns, epigenetic feature, specific Oct4 enhancer activity, and functional pluripotency. Oct4-GFPpositive EpiSCs present a higher degree of pluripotency, as they can contribute to chimera formation after blastocyst injection. Therefore, we hypothesized that Oct4-GFP-positive EpiSCs may have a higher reprogramming potential than Oct4- 
A

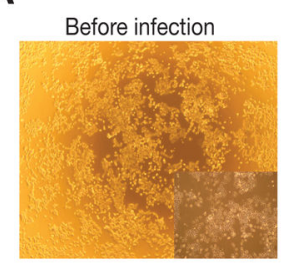

C

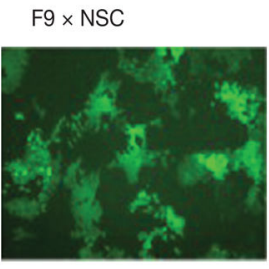

B

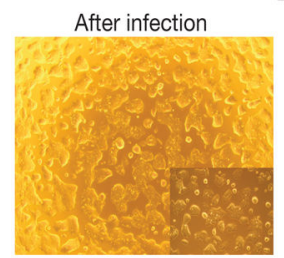

F9 $\times$ NSC

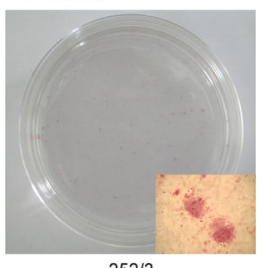

$252 / 3$

D

F9-Sox $2 \times N S C$
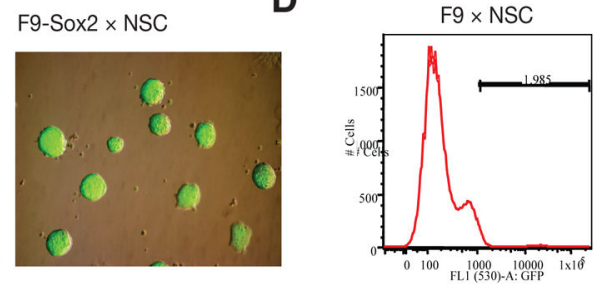

F9-Sox2 $\times$ NSC
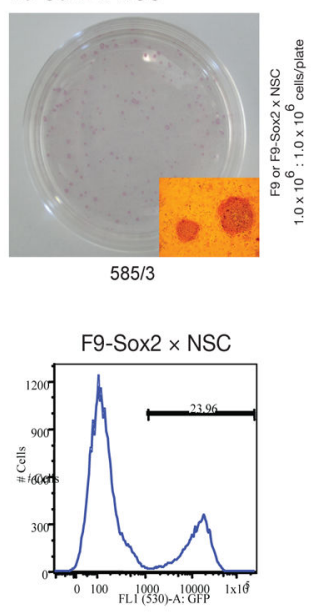

Figure 4. Role of Sox2 on the reprogramming potential of cells from a non-epiblast origin. (A) F9 ECCs overexpressing Sox2 exhibited a different morphology and presented more compact and round-shaped colonies. (B) The colony-forming rate was determined by AP staining after 1 week of neomycin selection. Data is represented as the number of total colonies from 3 different experiments. (C) Morphology of both F9 hybrid cells and F9-Sox2 hybrid cells. (D) The reprogramming rates of both F9 and F9Sox2 hybrids were calculated by counting the number of Oct4-GFP-positive cells via FACS.

doi: 10.1371/journal.pone.0067594.g004

GFP-negative EpiSCs. Following cell fusion, Oct4-GFPpositive cells produced an increased number of fusion hybrid colonies compared with control EpiSCs (Figure 5B). Again, these data show that cellular pluripotency is closely associated with reprogramming capability.

\section{Discussion}

In the current study, we attempted to uncover any association between the pluripotent state, naïve or primed, and the reprogramming capacity as well as the effect of Sox2 on the reprogramming potential. Although previous studies had demonstrated that the newly established EpiSCs exhibited cellular pluripotency [1,3], they had not determined the reprogramming potential of EpiSCs. To delve deeper into these issues, we fused NSCs with either ESCs or EpiSCs, and found that ESCs produced many more viable fusion hybrid colonies than EpiSCs. Likewise, P19 ECCs, which have an epiblast origin [13], also showed a delayed and a lower reprogramming potential than the non-epiblast-derived F9 ECCs. Thus, our results demonstrate that a low reprogramming potential is a general feature of mouse epiblast-derived pluripotent stem cells.

Both P19 ECCs and EpiSCs showed lower levels of SOX2 protein, but not OCT4, compared with F9 ECCs and ESCs, respectively. These findings indicate that although low Oct4 levels have a detrimental effect on the viability of pluripotent cells, low Sox2 levels have a moderate effect on the maintenance of pluripotency. Interestingly, Sox2 overexpression in P19 hybrid cells led to a significantly increased colony-forming rate and reprogramming efficiency, as well as to a shortened time frame for epigenetic modifications-activation of pluripotency marker genes, DNA demethylation of Oct4 regulatory regions, and X-chromosome reactivation. Although forced expression of Sox2 was described to trigger the differentiation of pluripotent cells [20], we observed that Sox2 overexpression induces compact colony morphology in F9 and P19 ECCs, as also previously reported [21]. EpiSCs overexpressing Sox2 produced significantly more fusion hybrids colonies compared with control EpiSCs. These results indicate that EpiSCs, along with P19 and F9 ECCs, could acquire a higher reprogramming capacity after Sox 2 overexpression.

Undifferentiated male EpiSCs should theoretically possess $\mathrm{X}$-chromosome patterns consistent with those of somatic cells. However, approximately half of the XY EpiSCs exhibit a pinpoint $X i s t / T$ six FISH signal, which is actually a feature of $X Y$ ESCs. EpiSCs are composed of two subpopulations of cells that represent the in-vivo early and late stage epiblast, as we have shown in our previous study [11]. Therefore, it is likely that EpiSCs with a pluripotent type of $X$ chromosome correspond to an in-vivo epiblast of an early stage, while those with a somatic type represent an in-vivo epiblast of a relatively late stage. It is also likely that the conversion of the pluripotent type of $X$ chromosome into the somatic type occurs faster in XX EpiSCs, which normally takes $24 \mathrm{hrs}$, as both $X X$ and $X Y$ EpiSCs originate from 5.5 d.p.c. embryos, which had $X$ chromosome inactivation nearly completed. Sox2 overexpression in EpiSCs also led to faster X-chromosome reactivation. While Epi-Sox2 hybrids showed nearly complete X-chromosome reactivation within 7 days post-fusion, more than $60 \%$ of control EpiSC hybrids showed remaining inactive $X$ chromosomes on day 7 post-fusion. In addition, the $X$ chromosome state of control EpiSC hybrids was consistent 
A

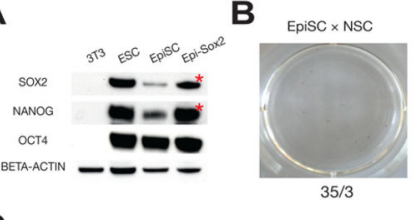

C

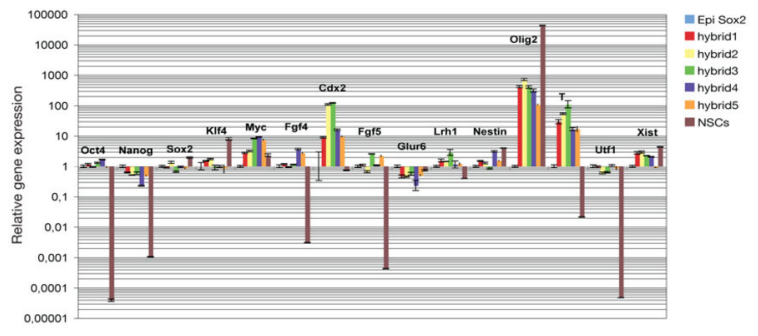

D

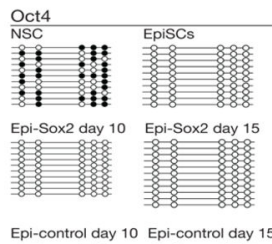

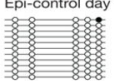

E
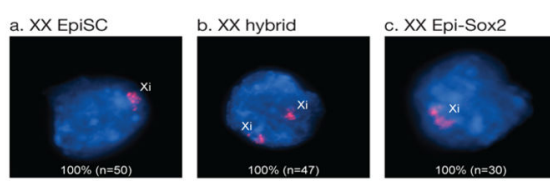

$\mathbf{F}$

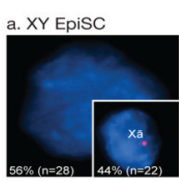

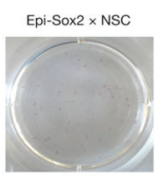

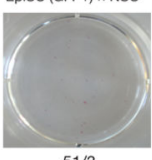

$51 / 3$
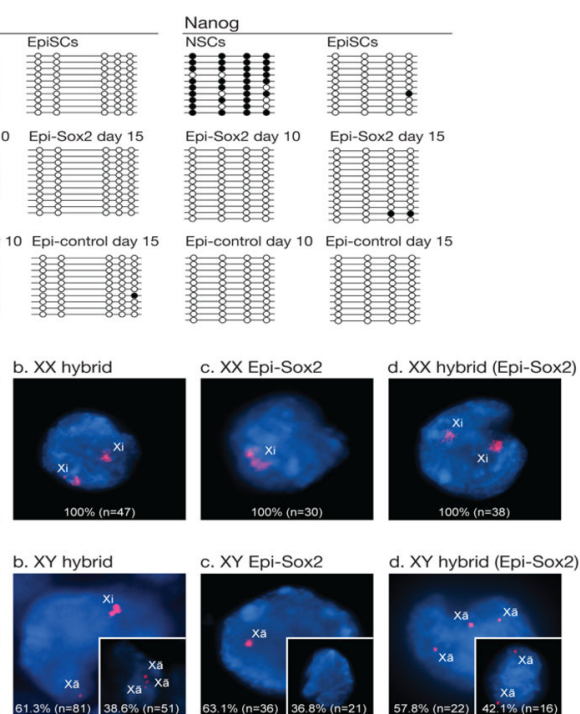

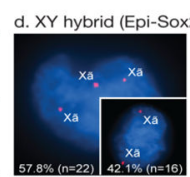

Figure 5. Sox2 overexpression rescues the low reprogramming potential of EpiSCs. (A) OCT4, NANOG, and SOX2 protein levels were determined in Sox2overexpressing EpiSCs (Epi-Sox2) by Western blot. (B) EpiSox2 showed increased colony-forming rates. Data is represented as the number of total colonies from 3 different experiments. (C) The expression profiles of pluripotency and NSC markers in Epi-Sox2 hybrids were assessed by real-time RT-PCR. All data are normalized to Bact expression and calibrated to EpiSCs, whose expression is considered 1 for all genes. The $y$-axis value is on logarithmic scale, and minor gridlines are $1 / 10$ th the value of each major gridline. Data is represented as mean +/- SEM. (D) DNA methylation state of Oct4 and Nanog promoter regions in control hybrids and EpiSox 2 hybrids was compared by bisulfite sequencing. Open and filled circles indicate unmethylated and methylated $\mathrm{CpGs}$, respectively. ( $E$ and $\mathbf{F}) \mathrm{X}$ chromosome state was determined by Xist/Tsix RNA FISH in XX (E) and XY (F) EpiSC hybrids and Epi-Sox2 hybrids on day 7 post-fusion. DNA was counterstained with DAPI (blue). Xist/Tsix RNA was detected as a large signal in $\mathrm{Xi}$ (inactive $\mathrm{X}$ chromosome), a pinpoint signal in Xã (active $X$ chromosome; pluripotent cell type), and no signal in Xa (active $\mathrm{X}$ chromosome; somatic cell type).

doi: 10.1371/journal.pone.0067594.g005 with a non-reprogrammed state as late as day 18 post-fusion (data not shown). Sox2 is known to bind on Xist and repress Xist expression together with Oct4 and Nanog [22]. Therefore, it is highly likely that EpiSCs acquire a higher X-chromosome reactivating potential after Sox2 overexpression.

P19 ECCs and EpiSCs express SOX2 and NANOG at lower levels than ESCs. A recent study [12] showed reversion of EpiSCs to an ESC-like state after Nanog overexpression. As Sox2 overexpression led to increased NANOG protein levels, we expected a Sox2-mediated reversion of EpiSCs into a naïve pluripotent state [12] but this did not occur (data not shown). Considering that Nanog is very sensitive to dosage levels $[12,15]$, it is very likely that the Nanog level indirectly induced via Sox2 overexpression is not sufficient to revert EpiSCs to an ESC-like state. Collectively, our findings suggest that Sox2 is a determinant of reprogramming capacity.

Cellular pluripotency can be determined by several criteria such as expression of pluripotency markers, in vitro differentiation potential, formation of teratomas and chimeric contribution into somatic cells and the germline. As EpiSCs with a primed pluripotency can form teratomas but not chimeras, the ability for chimeric contribution is a critical criterion for distinguishing a naïve and primed state of cellular pluripotency. However, the generation of mouse chimeras is not simple, as it requires highly experienced skills with a very complicated experimental setting. Based on our findings, ESCs and EpiSCs differ not only in chimeric contribution capacity, but also in reprogramming potential via fusion-mediated reprogramming. Therefore, our data suggest that the reprogramming potential of pluripotent stem cells could be a simple but effective indicator for distinguishing the functional state of pluripotent stem cells.

\section{Methods}

\section{Cell culture and fusion}

P19, F9 and 293T (ATCC) were cultured on gelatin-coated ( $0.1 \%$ in PBS) dishes in standard culture media: high-glucose DMEM (Gibco BRL) containing 15\% fetal calf serum (FCS; Gibco BRL), 1X penicillin/streptomycin/glutamine (Gibco BRL), and $1 \mathrm{X}$ nonessential amino acids (Gibco BRL). ESCs were grown in standard culture media plus LIF (Chemicon). The neural stem cell (NSC) line used in this study was derived from the brain tissue of 16.5-dpc OG2/ROSA26 female mice and has been previously reported [23]. NSCs were grown in DMEM-F12 medium (Gibco BRL) supplemented with $20 \mathrm{ng} / \mathrm{ml}$ epidermal growth factor (EGF; Peprotech), $20 \mathrm{ng} / \mathrm{ml} \mathrm{bFGF}$ (New England Biolabs), B27 supplement (Gibco BRL), $8 \mathrm{mM}$ HEPES (Gibco BRL) and 1X penicillin/streptomycin/glutamine. Feeder-free EpiSCs [23] were cultured in conditioned medium (CM). For conditioning, irradiated CF-1 mouse embryonic fibroblasts (MEFs) were seeded at density of $5 \times 10^{4}$ cells $/ \mathrm{cm}^{2}$ and incubated during $24 \mathrm{hrs}$ in Knockout DMEM medium (Invitrogen) containing 20\% Serum Replacement (Invitrogen), $1 \mathrm{X}$ penicillin/streptomycin/glutamine (Gibco BRL), 1X nonessential amino acids (Gibco BRL), 1X $\beta$-mercaptoethanol (Gibco BRL) and $5 \mathrm{ng} / \mathrm{ml}$ bFGF (New England Biolabs). The $\mathrm{CM}$ medium was filtered, and $\mathrm{bFGF}(5 \mathrm{ng} / \mathrm{ml})$ was added to it. 
For passaging feeder-free EpiSCs, colonies were incubated with Collagenase IV (Invitrogen) for $5 \mathrm{~min}$ at $37^{\circ} \mathrm{C}$ and triturated by using a cell scraper. Cell clumps were replated on FCScoated dishes, with medium changes every $24 \mathrm{hrs}$.

Pluripotent cells and NSCs were fused according to our previous protocol [14]. Briefly, pluripotent cells were mixed with NSCs in a 1:1 ratio, and then washed in PBS. The mixture was centrifuged at $130 \mathrm{~g}$ for $5 \mathrm{~min}$, and $1 \mathrm{ml}$ of a prewarmed $50 \%$ polyethylene glycol 1500 solution (PEG1500; Roche) was added to the cell pellet over $1 \mathrm{~min}$. An additional $20 \mathrm{ml}$ of DMEM was added to the cell suspension over $5 \mathrm{~min}$, with constant stirring. The cells were centrifuged at $130 \mathrm{~g}$ for $5 \mathrm{~min}$ to remove the PEG, washed gently with DMEM, and cultured in ECC, ESC, and EpiSC medium, respectively. The fusion hybrid cells were selected by treating neomycin $(300 \mu \mathrm{g} / \mathrm{ml})$ after 24 hrs post-fusion, and colony-forming rate was calculated by counting the number of colonies that stained positive for alkaline phosphate (AP, Alkaline Phosphatase Detection Kit, Chemicon) 1 week post-fusion.

\section{Western blot and immunocytochemistry}

Western blots were performed using anti-Nanog (Cosmo Bio, REC-RCAB0002PF, 1:500), anti-Oct4 (Abcam, ab19857, 1:400), and anti-Sox2 (Abcam, ab15830, 1:3000) primary antibodies. Anti-Sox2 (Chemicon, 2003600, 1:1000) and antiOct4 (Abcam, ab19857, 1:500) antibodies were used for immunocytochemistry.

\section{Xist/Tsix FISH (fluorescence in situ hybridization)}

Fusion hybrid cells were placed onto Roboz slides, which were then fixed in 4\% paraformaldehyde in PBS for $10 \mathrm{~min}$ at room temperature, rinsed in $70 \%$ ethanol, and stored in $70 \%$ ethanol at $4^{\circ} \mathrm{C}$ until use in FISH. Xist/Tsix RNA was detected with a Xist RNA FISH probe that was labelled with Cy3 and that spans the entire Xist cDNA. Xist/Tsix RNA FISH was performed as described previously [24]. Images were obtained using applied spectral imaging (ASI) camera and analyzed with FishView software (Applied Spectral Imaging; ASI GmBH).

\section{DNA methylation analysis}

To assess the DNA methylation status of Oct4 and Nanog regulatory elements, genomic DNA was treated with sodium bisulfite to convert all unmethylated cytosine residues into uracil ones using EpiTect Bisulfite Kit (Qiagen) according to manufacturer's protocol. Following bisulfite treatment, all the selected genomic regions were amplified using a nested primer approach. PCR amplifications were performed using SuperTaq polymerase (Ambion) in a total volume of $25 \mu \mathrm{l}$. All PCR amplifications consisted of a total of 40 cycles of denaturation at $94^{\circ} \mathrm{C}$ for $30 \mathrm{~s}$, annealing at the appropriate temperature for each target region for $30 \mathrm{~s}$, extension at $72^{\circ} \mathrm{C}$ for $30 \mathrm{~s}$ with a $1^{\text {st }}$ denaturation at $94^{\circ} \mathrm{C}$ for $5 \mathrm{~min}$, and a final extension at $72^{\circ} \mathrm{C}$ for $10 \mathrm{~min}$. The primer sequences and annealing temperatures used were as follows: endogenous Oct4 $1^{\text {st }}$ sense $5^{\prime}$ TTTGTTTTTTTATTTATTTAGGGGG-3', endogenous Oct4 $1^{\text {st }}$ antisense 5'- ATCCCCAATACCTCTAAACCTAATC-3' (299 bp, $\left.45^{\circ} \mathrm{C}\right)$, endogenous Oct4 $2^{\text {nd }}$ sense $5^{\prime}-$ GGGTTAGAGGTTAAGGTTAGAGGG -3', endogenous Oct4 $2^{\text {nd }}$ antisense 5'-CCCCCACCTAATAAAAATAAAAAAA -3' (161 bp, $\left.\quad 55^{\circ} \mathrm{C}\right)$, Nanog $1^{\text {st }}$ sense $5^{\prime}$ TTTGTAGGTGGGATTAATTGTGAA -3', Nanog $1^{\text {st }}$ antisense 5'-AAAAAATTTTAAACAACAACCAAAAA -3' (312 bp, 45 $\mathrm{C}$ ), Nanog $2^{\text {nd }}$ sense 5'- TTTGTAGGTGGGATTAATTGTGAA -3', and Nanog $2^{\text {nd }}$ antisense 5'AAAAAAACAAAACACCAACCAAAT -3' (188 bp, 55 C). For each primer set, $3 \mu$ of product from the first round of PCR was used in the second round of PCR. The amplified products were verified by electrophoresis on $1 \%$ agarose gel. The PCR products were subcloned using the PCR 2.1-TOPO vector (Invitrogen) according to the manufacturer's protocol. Reconstructed plasmids were purified using the QIAprep Spin Miniprep kit (Qiagen) and individual clones were sequenced (GATC-biotech, Germany). Clones were only accepted if there was at least $90 \%$ cytosine conversion and all possible clonalities were excluded based on the criteria from BiQ Analyzer software (Max Planck Society, Germany). For each fusion hybrid, results were confirmed by performing at least 10 replicates per selected genomic region and at least two separate bisulfite treatments.

\section{Vector construction and transduction with lentivirus vectors}

pLVTHM-Sox2 was constructed by replacing the GFP in pLVTHM with the Sox2 coding sequence that was directly amplified from ESCs. pLVTHM was provided by D. Trono (Geneva, Switzerland). The recombinant lentivirus was produced by transient transfection of 293T cells with $12 \mu \mathrm{g}$ of pLVTHM, $8.5 \mu \mathrm{g}$ of psPax2, and $3 \mu \mathrm{g}$ of pMD2.G using Lipofectamine 2000 (Invitrogen), according to the manufacturer's protocol. The supernatant was collected at 24 and $48 \mathrm{hrs}$ of transfection and was then concentrated by ultracentrifugation at $26,000 \mathrm{rpm}$ for $2 \mathrm{hrs}$ at $4^{\circ} \mathrm{C}$ using a SW41 rotor (Beckman Coulter). After ultracentrifugation, the supernatant was decanted, and the viral pellet was resuspended in $200 \mu \mathrm{l}$ of DMEM. The suspension was stored at $-80^{\circ} \mathrm{C}$ until use. Packaging plasmids were also provided by D. Trono. EpiSCs, P19 ECC and F9 ECC cells were plated on 24-well plates $\left(5 \times 10^{4}\right.$ cells/well), and after $24 \mathrm{hrs}, 20 \mu \mathrm{l}$ of the concentrated virus was added to the medium. Cells were washed after $16 \mathrm{hrs}$ of incubation. After $48 \mathrm{hrs}$, the cells were once again subjected to this procedure to increase the viral transduction.

\section{Flow cytometry}

For FACS sorting, cells were dissociated with $0.25 \%$ trypsin EDTA (Invitrogen), neutralized with DMEM containing $10 \%$ FCS, washed with PBS, and then filtered through a $40-\mu \mathrm{m}$ nylon mesh to remove large cell clusters. The cells were resuspended in the appropriate culture medium $\left(5 \times 10^{6}\right.$ cells $/ \mathrm{ml}$ ) and analyzed using a FACSAria cell sorter (BD Biosciences). GFP-positive cells were sorted by FACS using a 100- $\mu \mathrm{m}$ nozzle. 


\section{Supporting Information}

Figure S1. Overexpressing Sox2 in P19 ECCs. (A) The expression levels of Oct4, Nanog, and Sox2 were compared in three different cell lines: ESCs, F9 ECCs, and P19 ECCs. (B) Sox2 expression was analyzed after transduction of different amount of viruses into P19 ECCs. (C) Comparison of the morphology of P19 ECCs (non-infected) and P19-Sox2 cell lines (clone 1 3).

(TIF)

\section{References}

1. Brons IG, Smithers LE, Trotter MW, Rugg-Gunn P, Sun B et al. (2007) Derivation of pluripotent epiblast stem cells from mammalian embryos. Nature 448: 191-195. doi:10.1038/nature05950. PubMed: 17597762.

2. Nichols J, Smith A (2009) Naive and primed pluripotent states. Cell Stem Cell 4: 487-492. doi:10.1016/j.stem.2009.05.015. PubMed: 19497275.

3. Tesar PJ, Chenoweth JG, Brook FA, Davies TJ, Evans EP et al. (2007) New cell lines from mouse epiblast share defining features with human embryonic stem cells. Nature 448: 196-199. doi:10.1038/nature05972. PubMed: 17597760

4. Avilion AA, Nicolis SK, Pevny LH, Perez L, Vivian N et al. (2003) Multipotent cell lineages in early mouse development depend on SOX2 function. Genes Dev 17: 126-140. doi:10.1101/gad.224503. PubMed: 12514105.

5. Boyer LA, Lee TI, Cole MF, Johnstone SE, Levine SS et al. (2005) Core transcriptional regulatory circuitry in human embryonic stem cells. Cell 122: 947-956. doi:10.1016/j.cell.2005.08.020. PubMed: 16153702.

6. Chambers I, Silva J, Colby D, Nichols J, Nijmeijer B et al. (2007) Nanog safeguards pluripotency and mediates germline development. Nature 450: 1230-1234. doi:10.1038/nature06403. PubMed: 18097409.

7. Han DW, Greber B, Wu G, Tapia N, Araúzo-Bravo MJ et al. (2011) Direct reprogramming of fibroblasts into epiblast stem cells. Nat Cell Biol 13: 66-71. doi:10.1038/ncb2136. PubMed: 21131959.

8. Takahashi K, Yamanaka S (2006) Induction of pluripotent stem cells from mouse embryonic and adult fibroblast cultures by defined factors. Cell 126: 663-676. doi:10.1016/j.cell.2006.07.024. PubMed: 16904174.

9. Yu J, Vodyanik MA, Smuga-Otto K, Antosiewicz-Bourget J, Frane JL et al. (2007) Induced pluripotent stem cell lines derived from human somatic cells. Science 318: 1917-1920. doi:10.1126/science.1151526. PubMed: 18029452

10. McBurney MW (1993) P19 embryonal carcinoma cells. Int J Dev Biol 37: 135-140. PubMed: 8507558.

11. Han DW, Tapia N, Joo JY, Greber B, Araúzo-Bravo MJ et al. (2010) Epiblast stem cell subpopulations represent mouse embryos of distinct pregastrulation stages. Cell 143: 617-627. doi:10.1016/j.cell. 2010.10.015. PubMed: 21056461.

12. Silva J, Nichols J, Theunissen TW, Guo G, van Oosten AL et al. (2009) Nanog is the gateway to the pluripotent ground state. Cell 138: 722-737. doi:10.1016/j.cell.2009.07.039. PubMed: 19703398

13. Yeom YI, Fuhrmann G, Ovitt CE, Brehm A, Ohbo K et al. (1996) Germline regulatory element of Oct-4 specific for the totipotent cycle of embryonal cells. Development 122: 881-894. PubMed: 8631266.

\section{Acknowledgements}

We appreciate Kenjiro Adachi for the critical reading of the manuscript.

\section{Author Contributions}

Conceived and designed the experiments: DWH NT HRS. Performed the experiments: DWH NT KTL KPK KK HTL. Analyzed the data: DWH NT MJA KTL KPK KK HTL HRS. Wrote the manuscript: DWH NT HRS.

14. Han DW, Do JT, Gentile L, Stehling M, Lee HT et al. (2008) Pluripotential reprogramming of the somatic genome in hybrid cells occurs with the first cell cycle. Stem Cells 26: 445-454. doi:10.1634/ stemcells.2007-0553. PubMed: 18065396.

15. Silva J, Chambers I, Pollard S, Smith A (2006) Nanog promotes transfer of pluripotency after cell fusion. Nature 441: 997-1001. doi: 10.1038/nature04914. PubMed: 16791199.

16. Watanabe K, Ueno M, Kamiya D, Nishiyama A, Matsumura M et al. (2007) A ROCK inhibitor permits survival of dissociated human embryonic stem cells. Nat Biotechnol 25: 681-686. doi:10.1038/ nbt1310. PubMed: 17529971.

17. McBurney MW, Reuhl KR, Ally Al, Nasipuri S, Bell JC et al. (1988) Differentiation and maturation of embryonal carcinoma-derived neurons in cell culture. J Neurosci 8: 1063-1073. PubMed: 2894413.

18. Do JT, Han DW, Gentile L, Sobek-Klocke I, Wutz A et al. (2009) Reprogramming of Xist against the pluripotent state in fusion hybrids. J Cell Sci 122: 4122-4129. doi:10.1242/jcs.056119. PubMed: 19843582.

19. Do JT, Han DW, Gentile L, Sobek-Klocke I, Stehling M et al. (2008) Enhanced reprogramming of Xist by induced upregulation of Tsix and Dnmt3a. Stem Cells 26: 2821-2831. doi:10.1634/stemcells.2008-0482. PubMed: 18757295.

20. Kopp JL, Ormsbee BD, Desler M, Rizzino A (2008) Small increases in the level of Sox2 trigger the differentiation of mouse embryonic stem cells. Stem Cells 26: 903-911. doi:10.1634/stemcells.2007-0951. PubMed: 18238855

21. Zhao S, Nichols J, Smith AG, Li M (2004) SoxB transcription factors specify neuroectodermal lineage choice in ES cells. Mol Cell Neurosci 27: 332-342. doi:10.1016/j.mcn.2004.08.002. PubMed: 15519247.

22. Navarro $P$, Chambers I, Karwacki-Neisius V, Chureau C, Morey $C$ et al. (2008) Molecular coupling of Xist regulation and pluripotency. Science 321: 1693-1695. doi:10.1126/science.1160952. PubMed: 18802003.

23. Han DW, Do JT, Araúzo-Bravo MJ, Lee SH, Meissner A et al. (2009) Epigenetic hierarchy governing Nestin expression. Stem Cells 27: 1088-1097. doi:10.1002/stem.43. PubMed: 19415779.

24. Wutz A, Jaenisch $R$ (2000) A shift from reversible to irreversible $X$ inactivation is triggered during ES cell differentiation. Mol Cell 5: 695-705. doi:10.1016/S1097-2765(00)80248-8. PubMed: 10882105. 